\title{
Bounds on the recoverable deformations of polycrystalline SMAs at finite strain
}

\author{
Michaël Peigney ${ }^{1, a}$ \\ ${ }^{1}$ Univ Paris-Est, Laboratoire Navier (UMR 8205), CNRS, Ecole des Ponts ParisTech, IFSTTAR, F-77455 Marne la vallée, France
}

\begin{abstract}
This communication is concerned with the theoretical prediction of the recoverable strains (i.e. the strains that can be recovered by the shape memory effect) in polycrystalline SMAs. The analysis is carried out in the finite strain setting, considering a nonlinear elasticity model of phase transformation. The main results are some rigorous upper bounds on the set of recoverable strains. Those bounds depend on the polycrystalline texture through the volume fractions of the different orientations. A two-orientation polycrystal of tetragonal martensite is studied as an illustration. In that case, analytical expressions of the upper bounds are derived and the results are compared with lower bounds obtained by considering laminate textures. The issue of applying the proposed method to complex polycrystalline textures is commented on.
\end{abstract}

\section{Introduction}

A possible route to study the formation of microstructures in SMAs is to adopt a nonlinear elasticity model of phase transformation [1]. The general principle is that, under a prescribed loading, the system tends to minimize its free energy. Assuming the microscopic, mesoscopic and macroscopic scales to be well separated, the energy minimization principle leads to different expressions of the free energy at each scale. Denoting the microscopic free energy by $\Psi$, the mesoscopic energy $\tilde{\Psi}$ is obtained as the relaxation (or quasiconvex envelope) of $\Psi$, which essentially amounts to solve an optimal design problem with respect to the martensite/austenite geometric arrangement (see Sect. 2 for a precise definition). Viewing group of grains with the same orientation as individual homogeneous materials (governed by mesoscopic free energies), the polycrystal can be regarded as a composite material with a macroscopic energy $\bar{\Psi}$ obtained by homogenization of the constitutive free energy functions.

Assuming the microscopic free energy $\Psi$ to be known, determining its relaxation $\tilde{\Psi}$ largely remains an open problem. Estimating the macroscopic free energy $\bar{\Psi}$ is even more challenging as stress and strain compatibility conditions between the grains need to be taken into account. Of special interest are the strains that minimize the mesoscopic (resp. macroscopic) free-energy. Those energyminimizing strains can indeed be interpreted as the recoverable strains of a monocrystalline (resp. polycrystalline) shape memory alloy, i.e. the strains that can be recovered by the shape memory effect $[2,3]$. Knowing the set of recoverable strains is crucial for designing SMA systems. Experiments only give partial insight in the structure of that set, as they usually only give measurements

\footnotetext{
a e-mail: michael.peigney@polytechnique.org
}

along prescribed directions (see e.g. [4]). In this paper, we propose theoretical bounds on the whole set of recoverable strains, i.e. in the space of three-dimensional deformation gradients. Those bounds are expressed in terms of the lattice parameters and of statistical information on the polycrystalline texture (namely the orientation distribution function). Such data can be obtained experimentally using X-ray diffraction or EBSD (Electron Back Scattering Diffraction).

Most work related to that topic has been carried out in the geometrically linear setting, i.e. assuming small deformations with respect to a reference configuration $[2,5-$ 13]. In this paper, we focus on upper bounds of the recoverable strains of martensitic polycrystals, in the geometrically non-linear setting. The set of mesoscopic energyminimizing strains has been obtained in closed-form for a double-well energy [1]. Using known restrictions on Young measures $[1,14]$, an upper bound on the mesoscopic energy-minimizing strains has been proposed in the case of three or more wells [15]. Regarding polycrystals, a general method has been introduced in [16] for generating upper bounds on the set of macroscopic recoverable strains, assuming that the set of recoverable strains of the constitutive single crystals (or at least an upper bound on it) is known. The approach used in [16] is based on the translation method [17-19], which has proved to be a powerful tool in various problems related to homogenization [20-22]. In this communication, we first present in Sect. 2 the monocrystalline bound [15] and subsequently combine it with the methodology of [16] to derive explicit upper bounds for polycrystals (Sect. 3). It turns out, however, that the obtained bounds may fail to recover the single crystal bound in the homogeneous limit. Motivated by that observation, we modify the polycrystalline approach so as 
to take the special structure of the single crystal bound into account (Sect. 4). This results in new upper bounds for polycrystals, which improve on the bounds of Sect. 3 and are consistent with the single crystal bound in the homogeneous limit. A two-orientation/three-well polycrystal is studied as an illustrative example in Sect. 5.

\section{Single crystal}

In the framework of nonlinear elasticity at finite strains, the microscopic behavior of a shape-memory alloy is described by its free energy density $\Psi$, which is a function of the deformation gradient $F$. The principle of frame indifference implies that $\Psi(R . F)=\Psi(F)$ for any rotation $R$ and deformation gradient $F$. We denote by $\mathcal{K}$ the set of deformation gradients that minimize $\Psi$. Without loss of generality, we can assume that the minimum value of $\Psi$ is equal to 0 , so that $\Psi \geq 0$ and

$$
\mathcal{K}=\{F \mid \Psi(F)=0\} .
$$

If the temperature $T$ is below the transformation temperature $T^{0}$ (which is assumed throughout this paper), the set $\mathcal{K}$ takes the form

$$
\mathcal{K}=\bigcup_{i=1}^{m} \operatorname{SO}(3) U_{i}
$$

where $m$ is the number of martensitic variant and $U_{1}, \cdots, U_{m}$ are the transformations strains. The symmetric positive definite $U_{1}, \cdots, U_{m}$ are all symmetry related, i.e. for any $(i, j)$ there exists a rotation $R_{i j}$ such that $U_{j}={ }^{\mathrm{t}} R_{i j} \cdot U_{i} \cdot R_{i j}$ (here and in the following, the presuperscript ${ }^{\mathrm{t}}$ denotes the transpose operator). This is implies that $U_{1}, \cdots, U_{m}$ all have the same determinant $\eta$. It is convenient to introduce the set $\mathcal{E}$ defined as

$$
\mathcal{E}=\left\{F \in \mathbb{R}^{3 \times 3}: \operatorname{det} F=\eta\right\} .
$$

Consider a reference configuration where a domain $\Omega$ is occupied by a single crystal of shape memory alloy. The mesoscopic free energy of the single crystal is given by

$$
\tilde{\Psi}(\tilde{F})=\inf _{F \in \mathcal{A}(\tilde{F})}\langle\Psi(F)\rangle
$$

where $\langle$.$\rangle denotes volume average over the domain \Omega$ and the set $\mathcal{A}(\tilde{F})$ of admissible deformation gradient fields is defined by

$$
\begin{aligned}
\mathcal{A}(\tilde{F})= & \{F \mid \operatorname{det} F>0, \exists u(\boldsymbol{x}) \text { such that } \\
& F=\nabla u \text { in } \Omega ; u(\boldsymbol{x})=\tilde{F} \cdot \boldsymbol{x} \text { on } \partial \Omega\} .
\end{aligned}
$$

The function $\tilde{F} \mapsto \tilde{\Psi}(\tilde{F})$ is mathematically referred to as the quasiconvex envelope (or relaxation) of $\Psi$. This last denomination is justified by the fact that $\tilde{\Psi}$ is the largest function such that: (i) $\tilde{\Psi} \leq \Psi$, (ii) $\tilde{\Psi}$ and quasiconvex, i.e. satisfies

$$
\tilde{\Psi}(\tilde{F}) \leq\langle\tilde{\Psi}(F)\rangle \forall F \in \mathcal{A}(\tilde{F}) .
$$

Let $\tilde{\mathcal{K}}$ be the set of deformation gradients that minimize $\tilde{\Psi}$. Since $\tilde{\Psi}$ is positive and vanishes on $\mathcal{K}$, the minimum value of $\tilde{\Psi}$ is equal to 0 and we have

$$
\tilde{\mathcal{K}}=\{\tilde{F} \mid \tilde{\Psi}(\tilde{F})=0\} .
$$

The set $\tilde{\mathcal{K}}$ is also known as the quasiconvex hull of $\mathcal{K}$ $[14,23]$. The exact expression $\tilde{\mathcal{K}}$ remains generally out or reach. Therefore, bounds on $\tilde{\mathcal{K}}$ (in the sense of inclusion of sets) are of interest. In that regard, it can be proved [15] that the set $\tilde{\mathcal{K}}_{+}$defined by

$$
\begin{aligned}
& \tilde{\mathcal{K}}_{+}=\{\tilde{F} \in \mathcal{E}: \exists \theta \in \mathcal{T} \text { such that } \\
& \left.0 \geq \sup _{(a, b) \in \mathcal{C}}\left\{\Phi\left(\tilde{F} \cdot a+\tilde{F}^{*} \cdot b\right)-\sum_{i=1}^{m} \theta_{i} \Phi\left(U_{i} \cdot a+U_{i}^{*} \cdot b\right)\right\}\right\}
\end{aligned}
$$

is an upper bound on $\tilde{\mathcal{K}}$, i.e. satisfies $\tilde{\mathcal{K}} \subset \tilde{\mathcal{K}}_{+}$. In (4), the set $\mathcal{T}$ is defined by

$$
\mathcal{T}=\left\{\theta=\left(\theta_{1}, \cdots, \theta_{m}\right) \in \mathbb{R}^{m}: \theta_{i} \geq 0 ; \sum_{i=1}^{m} \theta_{i}=1\right\}
$$

and $\Phi$ is the frame indifferent function $\Phi: \mathbb{R}^{3 \times 3} \mapsto \mathbb{R}$ defined by

$$
\Phi(M)=\max _{R \in \mathrm{SO}(3)} \operatorname{tr}(R \cdot M)
$$

Note that

$$
\Phi(M)=\lambda_{3}+\lambda_{2}+\lambda_{1} \operatorname{sgn}(\operatorname{det} M)
$$

where $0 \leq \lambda_{1} \leq \lambda_{2} \leq \lambda_{3}$ are the eigenvalues of $\sqrt{{ }^{t} M \cdot M}$. The bound (4) is obtained using known restrictions on Young measures $[1,14]$. The crucial point is that the function $F \mapsto \Phi\left(F . a+F^{*} . b\right)$ is quasiconvex for all $a$ and $b$ [24].

In (4), $C$ is a given arbitrary subset of $\mathbb{R}^{3 \times 3} \times \mathbb{R}^{3 \times 3}$ : Each choice of $C$ generates a corresponding bound on $\tilde{\mathcal{K}}$. For a well chosen $C$, the bound given by (4) coincides with $\tilde{\mathcal{K}}$ for the reference cases where the exact expression of $\tilde{\mathcal{K}}$ is available (see [15]).

Finally, for a given $\tilde{F}$ in $\tilde{\mathcal{K}}_{+}$, we note that the vector $\theta$ in (4) can be interpreted as the volume fractions of the different wells in a microstructure realizing $\tilde{F}$.

\section{Polycrystal}

Now consider a polycrystal occupying a domain $\Omega$. We can decompose $\Omega$ as $\Omega=\cup_{r=1}^{n} \Omega^{r}$ where each sub-domain $\Omega^{r}$ is formed by grains with the same orientation. The microscopic free energy in $\Omega^{r}$ can be written as

$$
\Psi^{r}(F)=\Psi\left({ }^{\mathrm{t}} R^{r} \cdot F \cdot R^{r}\right)
$$

where $R^{r}$ is a rotation describing the orientation in $\Omega^{r}$ relative to a reference single crystal. Defining $\chi^{r}$ the characteristic function of $\Omega^{r}$ (i.e. $\chi^{r}(\boldsymbol{x})=1$ if $\boldsymbol{x} \in \Omega^{r}$, and $\chi^{r}(\boldsymbol{x})=0$ otherwise), the macroscopic free energy $\bar{\Psi}(\bar{F})$ of the polycrystal is given by

$$
\bar{\Psi}(\bar{F})=\min _{F \in \mathcal{A}(\bar{F})}\left\langle\sum_{r=1}^{n} \chi^{r} \tilde{\Psi}^{r}(F)\right\rangle
$$

where $\tilde{\Psi}^{r}$ is the relaxation of $\Psi^{r}$, as defined in (1) (see e.g.[3] for a detailed justification). In the following, we primarily focus on the set $\overline{\mathcal{K}}$ of deformation gradients that minimize the macroscopic energy, i.e.

$$
\overline{\mathcal{K}}=\{\bar{F} \mid \bar{\Psi}(\bar{F})=0\} .
$$


In view of (9), we have

$$
\overline{\mathcal{K}}=\{\bar{F} \mid \exists F \in \mathcal{A}(\bar{F}) ; F(\boldsymbol{x}) \in \tilde{\mathcal{K}}(\boldsymbol{x}) \text { for all } \boldsymbol{x} \in \Omega\} .
$$

where $\tilde{\mathcal{K}}(\boldsymbol{x})$ is the quasiconvex hull of

$$
\mathcal{K}(\boldsymbol{x})=\bigcup_{i=1}^{m} \operatorname{SO}(3) U_{i}(\boldsymbol{x})
$$

In (11), $U_{i}(\boldsymbol{x})$ is defined as $U_{i}(\boldsymbol{x})=\sum_{r=1}^{n} \chi^{r}(\boldsymbol{x}) U_{i}^{r}$ with $U_{i}^{r}=R^{r} . U_{i}{ }^{\mathrm{t}} R^{r}$. Eq. (10) shows that the distinctive properties of strains $\bar{F}$ in $\overline{\mathcal{K}}$ is that they can be realized by a deformation $u(\boldsymbol{x})$ whose gradient $F=\nabla u$ satisfies the local constraint $F(\boldsymbol{x}) \in \tilde{\mathcal{K}}(\boldsymbol{x})$ at each point.

An upper bound on $\overline{\mathcal{K}}$ that take one-point statistics of the functions $\chi^{r}$ has been derived [16]. With the present notations, that bound is characterized by

$$
\sup _{(a, b) \in C^{\prime}}\left\{\Phi\left(\bar{F} \cdot a+\bar{F}^{*} \cdot b\right)-\sum_{r=1}^{n}\left\langle\chi^{r}\right\rangle \sup _{F \in \tilde{\mathcal{K}}^{r}}\left\{\Phi\left(F \cdot a+F^{*} \cdot b\right)\right\}\right\} \leq 0 .
$$

where $C^{\prime}$ denote a given subset of $\mathbb{R}^{3 \times 3} \times \mathbb{R}^{3 \times 3}$ and $\tilde{\mathcal{K}}^{r}$ is the quasi convex hull of

$$
\mathcal{K}^{r}=\bigcup_{i=1}^{m} \operatorname{SO}(3) U_{i}^{r} \text {. }
$$

The upper bound in (12) has been used in [16] on some simple examples where the sets $\tilde{\mathcal{K}}^{r}$ are known. In more general situations, the direct application of the bound (12) is hampered by the fact that $\tilde{\mathcal{K}}^{r}$ is unknown. Such a difficulty can be overcome by using the results from Sect. 2 . Let indeed $\tilde{\mathcal{K}}_{+}^{r}$ be the upper bound of $\tilde{\mathcal{K}}^{r}$ defined in Eq. (4). Since $\tilde{\mathcal{K}}^{r} \subset \tilde{\mathcal{K}}_{+}^{r}$, we have

$$
\sup _{\tilde{\mathcal{K}}^{r}} \Phi\left(F . a+F^{*} \cdot b\right) \leq \sup _{\tilde{\mathcal{K}}_{+}^{r}} \Phi\left(F . a+F^{*} \cdot b\right) .
$$

Therefore, we obtain from (12) that any $\bar{F}$ in $\overline{\mathcal{K}}$ necessarily satisfies

$$
\sup _{(a, b) \in C^{\prime}}\left\{\Phi\left(\bar{F} \cdot a+\bar{F}^{*} \cdot b\right)-\sum_{r=1}^{n}\left\langle\chi^{r}\right\rangle \sup _{F \in \tilde{\mathcal{K}}_{+}^{r}}\left\{\Phi\left(F \cdot a+F^{*} \cdot b\right)\right\}\right\} \leq 0 .
$$

The calculation of the right-hand side in (13) can be further simplified if $C^{\prime}=C$, i.e. if the bound (13) and the bound $\tilde{\mathcal{K}}_{+}^{r}$ given by (4) are calculated using the same set of tensors $(a, b)$. In such case it can be verified that

$$
\sup _{F \in \tilde{\mathcal{K}}_{+}^{r}}\left\{\Phi\left(F . a+F^{*} . b\right)\right\}=\Phi_{i}^{r}(a, b)
$$

with $\Phi_{i}^{r}(a, b)=\Phi\left(U_{i}^{r} \cdot a+U_{i}^{r, *} \cdot b\right)$. We thus arrive at

$$
\overline{\mathcal{K}} \subset \overline{\mathcal{K}}_{+}^{0}
$$

where

$$
\begin{aligned}
\overline{\mathcal{K}}_{+}^{0}=\{\bar{F} \in \mathcal{E}: 0 \geq & \sup _{(a, b) \in \mathcal{C}}\left\{\Phi\left(\bar{F} \cdot a+\bar{F}^{*} \cdot b\right)\right. \\
& \left.\left.-\sum_{r=1}^{n}\left\langle\chi^{r}\right\rangle \max _{1 \leq i \leq m} \Phi_{i}^{r}(a, b)\right\}\right\} .
\end{aligned}
$$

The set $\overline{\mathcal{K}}_{+}^{0}$ is an explicit upper bound that depends on one-point statistics of the texture, i.e. on the volume fractions $\left\langle\chi^{r}\right\rangle$ of the different orientations. The set $\overline{\mathcal{K}}_{+}^{0}$ is defined by a set of nonlinear constraints on $\bar{F}$.

\section{Improved bound for polycrystals}

The bound $\overline{\mathcal{K}}_{+}^{0}$ in (15) can be improved upon by taking the special structure of the monocrystalline bound (4) into account, as is now explained. Consider a given $\bar{F}$ in $\overline{\mathcal{K}}$. By (10), there exists a field $F \in \mathcal{A}(\bar{F})$ such that $F(\boldsymbol{x}) \in \tilde{\mathcal{K}}(\boldsymbol{x})$ for all $\boldsymbol{x} \in \Omega$. Using the bound (4) on $\tilde{\mathcal{K}}(\boldsymbol{x})$, we know there exists $\theta(\boldsymbol{x}) \in \mathcal{T}$ such that

$0 \geq \Phi\left(F(\boldsymbol{x}) \cdot a+F^{*}(\boldsymbol{x}) \cdot b\right)-\sum_{i=1}^{m} \theta_{i}(\boldsymbol{x}) \Phi\left(U_{i}(\boldsymbol{x}) \cdot a+U_{i}^{*}(\boldsymbol{x}) \cdot b\right)$

for all $a$ and $b$. Since $\chi^{r}(\boldsymbol{x}) \in\{0,1\}$ and $\sum_{r} \chi^{r}(\boldsymbol{x})=1$, Eq. (16) can be rewritten as

$$
0 \geq \Phi\left(F(\boldsymbol{x}) \cdot a+F^{*}(\boldsymbol{x}) \cdot b\right)-\sum_{r=1}^{n} \sum_{i=1}^{m} \chi^{r}(\boldsymbol{x}) \theta_{i}(\boldsymbol{x}) \Phi_{i}^{r}(a, b)
$$

For any $r=1, \cdots, n$ and $i=1, \cdots, m$, define

$$
\theta_{i}^{r}(\boldsymbol{x})=\chi^{r}(\boldsymbol{x}) \theta_{i}(\boldsymbol{x})
$$

Taking volume averages in (17) yields

$$
0 \geq\left\langle\Phi\left(F . a+F^{*} . b\right)\right\rangle-\sum_{r=1}^{n} \sum_{i=1}^{m}\left\langle\theta_{i}^{r}\right\rangle \Phi_{i}^{r}(a, b)
$$

for all $a$ and $b$. The crucial point is that the function $F \mapsto \Phi\left(F . a+F^{*} . b\right)$ is quasiconvex, which in view of (19) implies that

$$
0 \geq \Phi\left(\bar{F} . a+\bar{F}^{*} . b\right)-\sum_{r=1}^{n} \sum_{i=1}^{m}\left\langle\theta_{i}^{r}\right\rangle \Phi_{i}^{r}(a, b)
$$

for all $a$ and $b$. The scalar $\left\langle\theta_{i}^{r}\right\rangle$ can be interpreted as the volume fraction of martensitic variant $i$ with orientation $r$. Note from (18) that $\left\{\left\langle\theta_{i}^{r}\right\rangle\right\}_{1 \leq i \leq m}^{1 \leq r \leq n}$ belongs to the set $\overline{\mathcal{T}}$ defined by

$$
\overline{\mathcal{T}}=\left\{\Theta \in \mathbb{R}_{m}^{n} \mid \Theta_{i}^{r} \geq 0 ; \sum_{i=1}^{m} \Theta_{i}^{r}=\left\langle\chi^{r}\right\rangle \forall r=1, \cdots, n\right\} .
$$

The developments so far show that for any $\bar{F}$ in $\overline{\mathcal{K}}$, there exists $\boldsymbol{\Theta} \in \overline{\mathcal{T}}$ verifying the inequality (20). This last statement can be rewritten as

$$
\overline{\mathcal{K}} \subset \overline{\mathcal{K}}_{+}
$$

where

$$
\begin{aligned}
\overline{\mathcal{K}}_{+} \quad & =\{\bar{F} \in \mathcal{E}: \exists \Theta \in \overline{\mathcal{T}} \text { such that } \\
& 0 \geq \sup _{(a, b) \in \mathcal{C}}\left\{\Phi\left(\bar{F} \cdot a+\bar{F}^{*} \cdot b\right)-\sum_{r=1}^{n} \sum_{i=1}^{m} \Theta_{i}^{r} \Phi_{i}^{r}(a, b)\right\} .
\end{aligned}
$$


In a way similar to the bound $\overline{\mathcal{K}}_{+}^{0}$ considered in Sect. 3 , $\overline{\mathcal{K}}_{+}$depends on the texture through the volume fractions $\left\langle\chi^{r}\right\rangle$ of the different orientations (the later indeed appear in the definition (21) of the set $\overline{\mathcal{T}})$. by

For a given $\bar{F}$ in $\mathcal{E}$, let $Q(\bar{F})$ be the subset of $\mathbb{R}_{m}^{n}$ defined

$$
\begin{aligned}
Q(\bar{F}) & =\{\Theta \in \overline{\mathcal{T}}: \\
& \left.0 \geq \sup _{(a, b) \in \mathcal{C}}\left\{\Phi\left(\bar{F} \cdot a+\bar{F}^{*} \cdot b\right)-\sum_{r, i} \Theta_{i}^{r} \Phi_{i}^{r}(a, b)\right\}\right\}
\end{aligned}
$$

Observe that $Q(\bar{F})$ is a convex set defined by a family of linear constraints. The set $Q(\bar{F})$ can be interpreted as the set of volume fractions in the microstructures realizing $\bar{F}$. The distinctive propery of strains $\bar{F}$ in $\overline{\mathcal{K}}_{+}$is that $Q(\bar{F})$ is non empty. In the language of linear programming, checking whether the convex set $Q(\bar{F})$ is non empty amounts to check feasibility of the linear constraints in (24) [25], which is not a direct calculation - even for a discrete $C$. In that regard, it can be noted that interior-point methods [26] offer some efficient algorithms for detecting feasibility in large-scale linear programming problems. Interestingly, such algorithms, as the self-dual algorithm of Ye [27], have been used in other problems related to shapememory alloys [28] and could possibly be useful for calculating the bound $\overline{\mathcal{K}}_{+}$in the case of a complex polycrystalline texture.

\section{Illustrative example}

\subsection{Upper bounds}

We consider a polycrystal with two orientations, assuming without loss of generality that orientation 1 is the reference orientation. The constitutive single crystals obey a cubic to tetragonal transformation: We have $\mathcal{K}^{1}=\bigcup_{i=1}^{3} \operatorname{SO}(3) U_{i}^{1}$ where

$$
\begin{gathered}
U_{1}^{1}=\left(\begin{array}{ccc}
\eta_{2} & 0 & 0 \\
0 & \eta_{1} & 0 \\
0 & 0 & \eta_{1}
\end{array}\right), U_{2}^{1}=\left(\begin{array}{ccc}
\eta_{1} & 0 & 0 \\
0 & \eta_{2} & 0 \\
0 & 0 & \eta_{1}
\end{array}\right), \\
U_{3}^{1}=\left(\begin{array}{ccc}
\eta_{1} & 0 & 0 \\
0 & \eta_{1} & 0 \\
0 & 0 & \eta_{2}
\end{array}\right) .
\end{gathered}
$$

These matrix representations are relative to the reference orthonormal basis $\left(v_{1}, v_{2}, v_{3}\right)$ of the cubic austenitic lattice in orientation 1. All the results presented next are obtained with the lattice parameters of $\mathrm{MnCu}$, i.e. $\eta_{1}=1.0099$, $\eta_{2}=0.9656$ [29]. The set $\mathcal{K}^{2}$ of strains that minimize the microscopic free energy in orientation 2 can be written as $\mathcal{K}^{2}=R^{2} \cdot \mathcal{K}^{1} \cdot{ }^{\mathrm{t}} R^{2}$ where $R^{2}$ is the rotation taken as

$$
R^{2}=\left(\begin{array}{rrr}
\frac{\sqrt{2}}{2} & -\frac{\sqrt{2}}{2} & 0 \\
\frac{\sqrt{2}}{2} & \frac{\sqrt{2}}{2} & 0 \\
0 & 0 & 1
\end{array}\right)
$$

Textures satisfying the assumptions made so far (i.e. $n=2$ with $R^{1}=I$ and $R^{2}$ given by Eq. (25) ) are observed in some ribbons of shape memory alloys [30].

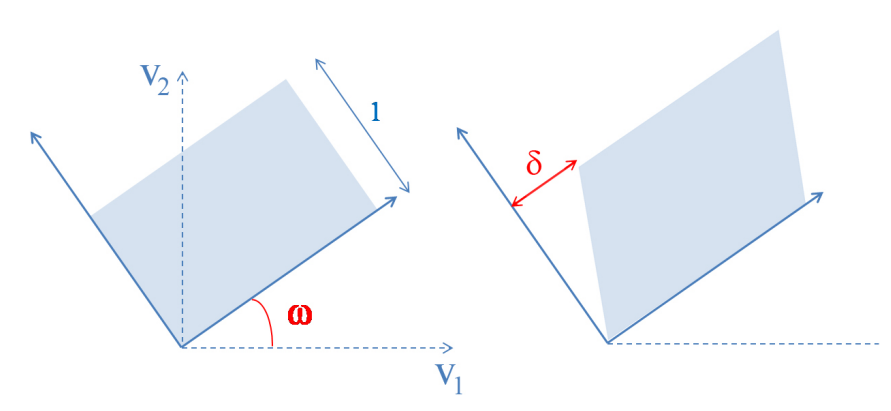

Figure 1. Representation of the deformation $\boldsymbol{I}+\delta u(\omega) \otimes v(\omega)$ : reference (left) and deformed (right) configuration.

Consider deformation gradients $F(\omega, \delta)$ of the form

$$
F(\omega, \delta)=\left(\eta_{1}^{2} \eta_{2}\right)^{1 / 3}(\boldsymbol{I}+\delta u(\omega) \otimes v(\omega))
$$

where

$u(\omega)=\cos \omega v_{1}+\sin \omega v_{2}$ and $v(\omega)=-\sin \omega v_{1}+\cos \omega v_{2}$

The deformation gradient $F(\omega, \delta)$ is a simple shear between the directions $u(\omega)$ and $v(\omega)$ (Figure 1), followed by a uniform dilatation $\left(\eta_{1}^{2} \eta_{2}\right)^{1 / 3} \boldsymbol{I}$. The parameter $\omega$ is the angle made by the shear directions $(u(\omega), v(\omega))$ with the directions $\left(v_{1}, v_{2}\right)$ of the cubic austenitic lattice in orientation 1 .

The results of Sect. 3-4 allow one to bound the values $(\omega, \delta)$ for which $F(\omega, \delta)$ is recoverable. The solid lines in Fig. 2 shows the boundary of the domain

$$
\Delta_{+}=\left\{(\omega, \delta): F(\omega, \delta) \in \overline{\mathcal{K}}_{+}\right\}
$$

where the bound $\overline{\mathcal{K}}_{+}$is calculated using (23) with a well chosen class $C$ of tensors $(a, b)$ for which closed-form expressions can be obtained (see [24] for details). The volume fraction $\left\langle\chi^{1}\right\rangle$ is set equal to 0.7 . Any recoverable deformation gradient $F(\omega, \delta)$ is necessarily within the bounded domain $\Delta_{+}$delimited by the solid lines in Figures 2.

Similarly, the dotted lines in Fig. 2 show the boundary of the domain

$$
\Delta_{+}^{0}=\left\{(\omega, \delta): F(\omega, \delta) \in \overline{\mathcal{K}}_{+}^{0}\right\}
$$

where $\overline{\mathcal{K}}_{+}^{0}$ is calculated using the same tensors $(a, b)$ as for the bound $\overline{\mathcal{K}}_{+}$. This allows one to appreciate the improvement brought by the consideration of (23) over (15).

\subsection{Lower bound}

The relations defining $\overline{\mathcal{K}}_{+}$in $(23)$ are necessary- but not sufficient - conditions for a deformation gradient to be recoverable. The issue is to determine which deformation gradients in $\overline{\mathcal{K}}_{+}$are indeed recoverable for some polycrystalline texture that is compatible with the prescribed statistics (i.e. with prescribed volume fractions of the different orientations). Considering the special class of laminated textures and adapting an argument introduced in [1], a set 


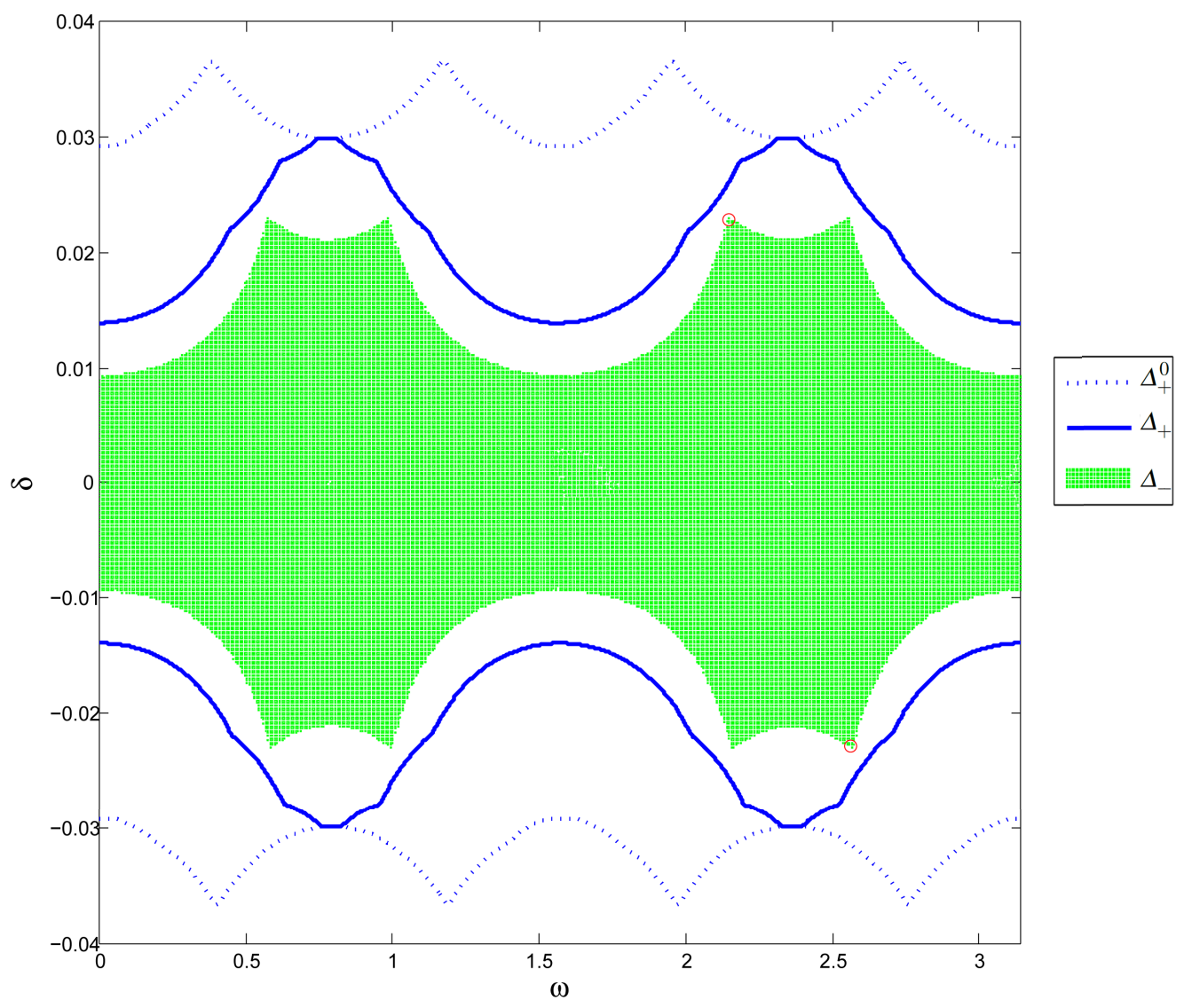

Figure 2. Bounds on the shear $\delta$ for $\mathrm{MnCu},\left\langle\chi^{1}\right\rangle=0.7$.

of values $\left(\omega^{*}, \delta^{*}\right)$ for which $F\left(\omega^{*}, \delta^{*}\right)$ is recoverable can be constructed. That set is denoted by $\Delta_{-}$and shown in green in Figure 2. The green domain $\Delta_{-}$is found to fill most of the domain $\Delta_{+}$, meaning that most of the values of $(\omega, \delta)$ in $\Delta_{+}$can be realized by laminate textures. The gap between $\Delta_{-}$and $\Delta_{+}$could possibly be reduced by considering more complex polycrystalline textures.

\section{Concluding remarks}

In this paper, some rigorous upper bounds on the recoverable strains of martensitic polycrystals have been obtained in the geometrically nonlinear setting. The main results are the bounds $\overline{\mathcal{K}}_{+}^{0}$ and $\overline{\mathcal{K}}_{+}$(defined in (15) and (23) respectively) that depend on the texture through the volume fractions of the different orientations. Those bounds are expressed in terms of a given family $C$ of tensors $(a, b)$, which acts as a free parameter in (15)-(23): each choice of $C$ generates corresponding bounds $\overline{\mathcal{K}}_{+}^{0}$ and $\overline{\mathcal{K}}_{+}$. For a given (say discrete) $\mathcal{C}$, the bound $\overline{\mathcal{K}}_{+}$is tighter than $\overline{\mathcal{K}}_{+}^{0}$ but more difficult to calculate: whereas checking if a given deformation gradient $\bar{F}$ is in $\overline{\mathcal{K}}_{+}^{0}$ is a direct calculation, checking if $\bar{F} \in \overline{\mathcal{K}}_{+}$amounts to detecting feasibility of a linear programming problem in $\mathbb{R}_{m}^{n}$. Those bounds could be evaluated in closed form for the 2-orientation/3-variant polycrystal presented as an illustrative example. For more complex textures, it is clear that numerical calculations of the bounds will be necessary at some point, which requires adequate algorithms, as discussed in Sect. 4. A more theoretical line of investigation consists in deriving upper bounds taking more information on the texture (such as 2-point statistics) into account.

\section{References}

[1] J. Ball, R. James, Phil. Trans. Roy. Soc. London A. 338, 338 (1992)

[2] K. Bhattacharya, R. Kohn, Arch. Rational Mech. Anal. 139, 99 (1997)

[3] K. Bhattacharya, Microstructure of Martensite (Oxford University Press, 2003)

[4] L. Zhao, P. Willemse, J. Mulder, J. Beyer, W. Wei, Scripta Mater. 39, 1317 (1998)

[5] R. Kohn, Continuum Mech. Thermodyn. 3, 193 (1991)

[6] A.C. Pipkin, The Quarterly Journal of Mechanics and Applied Mathematics 44, 1 (1991)

[7] V. Smyshlyaev, J. Willis, Proc. R. Soc. Lond. A 455, 779 (1998)

[8] S. Govindjee, A. Mielke, G.J. Hall, J. Mech. Phys. Solids 51, I (2003)

[9] S. Govindjee, K. Hackl, R. Heinen, Continuum Mech. Thermodyn. 18, 443 (2007)

[10] M. Peigney, J. Mech. Phys. Solids 57, 970 (2009) 
[11] I.V. Chenchiah, A. Schlömerkemper, Arch. Rational Mech. Anal. 207, 39 (2013)

[12] M. Peigney, J.Mech.Phys.Solids 61, 1511 (2013)

[13] Y. Shu, K. Bhattacharya, Acta Mater. 15, 5457 (1998)

[14] S. Müller, in Calculus of variations and geometric evolution problems (Springer, 1999)

[15] M. Peigney, J.Mech.Phys.Solids 61, 1489 (2013)

[16] M. Peigney, J. Mech. Phys. Solids 56, 360 (2008)

[17] K. Lurie, A. Cherkaev, Proc. Royal Soc. Edin A 99, 71 (1984)

[18] F. Murat, L. Tartar, in Les méthodes de l'homogénéisation: théorie et applications en physique (1985), Vol. 57, pp. 319-369

[19] G. Milton, The theory of composites (Cambridge University Press, 2004)

[20] G. Milton, S. Serkov, J. Mech. Phys. Solids 48, 1295 (2000)
[21] D. Talbot, J. Willis, Proc. R. Soc. Lond. A 460, 2705 (2004)

[22] M. Peigney, J. Mech. Phys. Solids 53, 923 (2005)

[23] B. Dacorogna, Direct methods in the calculus of variations, second edition (Springer, 2008)

[24] M. Peigney, Continuum Mech. Thermodyn. pp. 124,DOI 10.1007/s00161-015-0427-4 (2015)

[25] Y. Ye, Interior point algorithms: theory and analysis, Wiley Interscience (1997)

[26] S.J. Wright, Primal-dual interior-point methods (Siam, 1997)

[27] Y. Ye, Mathematical Programming 76, 211 (1997)

[28] M. Peigney, J. Seguin, E. Hervé-Luanco, Int. J. Sol. Struct. 48, 2791 (2011)

[29] Z. Basinski, J. Christian, Journal of the Institute of Metals 80, 659 (1952)

[30] S. Eucken, J. Hirsch, The effect of textures on shape memory behaviour, in Materials Science Forum (Trans Tech Publ, 1991), Vol. 56, pp. 487-492 